\title{
Neoplasias encefálicas em 40 cães: aspectos clínico-epidemiológicos e patológicos ${ }^{1}$
}

\author{
Rafael O. Chaves ${ }^{2 *}$, João P.S. Feranti³ ${ }^{3}$ Bruna Copat ${ }^{4}$, Angel Ripplinger ${ }^{3}$, \\ Raqueli T. França ${ }^{2}$, Glaucia D. Kommers ${ }^{5}$, Rafael A. Fighera ${ }^{5}$ e Alexandre Mazzanti ${ }^{6}$
}

\begin{abstract}
Chaves R.O., Feranti J.P.S., Copat B., Ripplinger A., França R.T., Kommers G.D., Fighera R.A. \& Mazzanti A. 2018. [Brain neoplasms in 40 dogs: clinical, epidemiological and pathological aspects]. Neoplasias encefálicas em 40 cães: aspectos clínico-epidemiológicos e patológicos. Pesquisa Veterinária Brasileira 38(4)734-740. Departamento de Clínica de Pequenos Animais, Universidade Federal de Santa Maria, Av. Roraima 1000, Santa Maria, RS 97105-900, Brazil. E-mail: rafaelochaves@hotmail.com

A retrospective study including dogs with brain neoplasms was conducted at the Service of Neurology (SN) of the Veterinary Teaching Hospital, from 2006 to 2015, with the objective to identify and characterize breed, sex, age, neurological signs, the location, the clinical evolution, the type and origin of the tumor and the findings of complementary examinations and necropsy. Of the 40 dogs with brain tumors included in this study $67.5 \%$ were classified as primary origin. Mixed breed dogs and Boxers were the most affected. The main clinical signs observed included seizures, behavioral changes and walk in circle. The thalamus-cortex region was the most affected. Regarding the type of the tumor, the meningioma (32.5\%) and oligodendroglioma (12.5\%) were the most common. The evolution of the clinical signs varied from four to 210 days (mean 44 days).
\end{abstract}

INDEX TERMS: Brain, neoplasms, dogs, neurology, intracranial tumors, pathology, clinics.

RESUMO.- Foi realizado um estudo retrospectivo de cães atendidos no Serviço de Neurologia (SN) do Hospital Veterinário Universitário (HVU), de 2006 a 2015, com o objetivo de identificar cães com neoplasias encefálicas e obter informações a respeito da raça, do sexo, da idade, dos sinais neurológicos, da localização, da evolução clínica, do tipo e origem do tumor e dos achados de exames complementares e de necropsia. Dos 40 cães com neoplasias encefálicas incluídos neste estudo, $67,5 \%$ foram classificadas como de origem primária. Cães sem raça definida e Boxers foram os mais acometidos. Os principais sinais clínicos observados incluíram crise

\footnotetext{
${ }^{1}$ Recebido em 4 de março de 2017.

Aceito para publicação em 31 de março de 2017.

${ }^{2}$ Curso de Medicina Veterinária, Centro de Ciências Biológicas e da Saúde, Universidade de Caxias do Sul (UCS), Bairro Petrópolis, Caxias do Sul, RS 95070-560, Brasil.*Corresponding author: rafaelochaves@hotmail.com

${ }^{3}$ Programa de Pós-Graduação em Medicina Veterinária, área de concentração em Cirurgia, Centro de Ciências Rurais (CCR), Universidade Federal de Santa Maria (UFSM), Camobi, Santa Maria, RS 97105-900, Brasil.

${ }^{4}$ Programa de Residência do Curso de Medicina Veterinária, Universidade Luterana do Brasil (ULBRA), Bairro São José, Canoas, RS 92425-900, Brasil.

${ }^{5}$ Departamento de Patologia, Centro de Ciências da Saúde, Universidade Federal de Santa Maria (UFSM), Santa Maria, RS 97105-900.

${ }^{6}$ Departamento de Clínica de Pequenos Animais, Centro de Ciências Rurais (CCR), Universidade Federal de Santa Maria (UFSM), Santa Maria, RS 97105-900.
}

epiléptica, alteração de comportamento e andar em círculo. A região tálamo-cortical foi a mais afetada. Quanto ao tipo do tumor, o meningioma $(32,5 \%)$ e o oligodendroglioma $(12,5 \%)$ foram os mais encontrados. A evolução dos sinais clínicos variou entre quatro e 210 dias (média de 44 dias).

TERMOS DE INDEXAÇÃO: Neoplasias, encéfalo, doenças de cães, caninos, neurologia, tumores intracranianos, patologia, clínica.

\section{INTRODUÇÃO}

Neoplasias intracranianas representam uma causa frequente de disfunção neurológica em animais de companhia de meia idade a idosos (Costa 2009, McEntee \& Dewey 2013). 0 diagnóstico para essa afecção vem aumentando nos últimos tempos com a utilização de técnicas de imagens avançadas, como a tomografia computadorizada e a ressonância magnética. Porém, como ambos os procedimentos não são realizados de forma rotineira em nosso país na maior parte das clínicas e hospitais veterinários, existe a impressão de raridade (Costa 2009). As neoplasias encefálicas primárias incluem os que se originam no parênquima encefálico (células da glia e neurônio), nas células da camada interna e externa do encéfalo (plexo coroide) e em elementos vasculares. As de origem secundária (menos comum, incluem neoplasias metastáticas 
e tumores localizados fora do encéfalo e que se estendem até ele comprimindo as estruturas intracranianas (Costa 2009, McEntee \& Dewey 2013, Song et al. 2013, Dewey 2016).

Para Bagley et al. (1999), Costa (2009) e Song et al. (2013), as neoplasias encefálicas demonstram predileção racial e as mais afetadas incluem Golden retriever, Boxer, Labrador retriever, Boston terrier, Bulldog francês, Collie e Schnauzer (Sturges et al. 2008, O’Brien \& Coates 2010, Song et al. 2013, Dewey 2016). Geralmente afetam a região tálamo-cortical (Sturges et al. 2008, Da Costa 2009), mas também podem envolver mais de uma região anatômica do encéfalo (Snyder et al. 2006, Snyder et al. 2008, Sturges et al. 2008, Costa 2009).

Os sinais neurológicos dependem da localização, do tamanho e da taxa de crescimento do tumor (Costa 2009, Dewey 2016). Esses sinais são resultantes de compressão de estruturas adjacentes, invasão direta dos tecidos, interrupção da circulação, edema, inflamação e necrose (Costa 2009, O’Brien \& Coates 2010, Rossmeisl \& Pancotto 2012, Dewey 2016). Menos comum, no entanto, os sinais clínicos também podem ser multifocais dependendo do número e da localização das massas tumorais e das alterações locais provocadas (Snyder et al. 2006, Snyder et al. 2008, Sturges et al. 2008, Costa 2009).

O diagnóstico presuntivo de neoplasia encefálica pode ser estabelecido de acordo com o histórico, a raça, a idade, os sinais neurológicos, a evolução dos sinais e os resultados de exames complementares (radiografia torácica, ultrassonografia abdominal, análise do líquido cerebroespinhal (LCE), tomografia computadorizada e ressonância magnética). Por outro lado, o diagnóstico definitivo é estabelecido pela análise histopatológica, realizada por meio de biópsia ou necropsia (Costa 2009, O’Brien \& Coates 2010, Ródenas et al. 2011, Rossmeisl \& Pancotto 2012, Dewey 2016).

Vários estudos retrospectivos sobre neoplasias encefálicas são encontrados na literatura, basicamente internacional (Kraft et al. 1997, Bley et al. 2005, Dickinson et al. 2006, Montoliu et al. 2006, Snyder et al. 2006, Snyder et al. 2008, Sturges et al. 2008, Ródenas et al. 2011, Song et al. 2013). Na literatura nacional, entretanto, existem poucos dados epidemiológicos acerca do tema (Santos et al. 2012, Marcasso et al. 2015) que demonstrem os principais tumores do encéfalo de cães e as principais alterações encontradas. Com base nisso, o objetivo deste estudo retrospectivo foi identificar cães com neoplasias encefálicas, atendidos no Hospital Veterinário Universitário (HVU) da UFSM, entre janeiro de 2006 e dezembro de 2015, e obter informações a respeito da raça, do sexo, da idade, dos sinais neurológicos, da localização, da evolução clínica, do tipo e origem do tumor e dos achados de exames complementares e de necropsia.

\section{MATERIAL E MÉTODOS}

Foram revisados os arquivos do Serviço de Neurologia do HVU-UFSM na procura de casos de neoplasias envolvendo o encéfalo de cães, entre janeiro de 2006 e dezembro de 2015. Incluíram-se neste estudo, somente cães que apresentavam dados completos de história clínica, sinais clínicos, exames neurológicos e complementares e com diagnóstico definitivo confirmado pela necropsia e exame histopatológico, realizados no Laboratório de Patologia Veterinária (LPV-UFSM). Dos 745 casos neurológicos confirmados, envolvendo o sistema nervoso central, atendidos no período do estudo, 50 cães apresentavam neoplasia encefálica, destes, somente 40 foram incluídos de acordo com os critérios pré-estabelecidos. 0 exame neurológico foi constituído de observações do estado mental (comportamento e consciência), avaliação da postura e locomoção, das reações posturais (posicionamento proprioceptivo e teste do salto), dos nervos cranianos, dos reflexos segmentares espinhais e da palpação epaxial (hiperestesia) da região cervical (Dewey et al. 2016b). A localização das lesões foi estabelecida de acordo com os sinais neurológicos apresentados pelos pacientes. A divisão neuroanatômica da lesão foi definida em tálamo-córtex, tronco encefálico e cerebelo. Quando a neoplasia envolvia mais de uma região anatômica foi classificada como multifocal. Em todos os casos foram realizados métodos complementares de diagnóstico, que incluíram: hemograma, bioquímica sérica (determinação de ureia, creatinina, glicose, alanina aminotransferase (ALT), fosfatase alcalina (FA), proteína sérica total e albumina) e urinálise. Em alguns pacientes foi realizada análise de LCE, mediante punção na região lombar (L5-L6). Os exames de imagem utilizados incluíram radiografia simples de tórax e ultrassonografia abdominal. Foram consideradas neoplasias primárias aquelas originadas do parênquima encefálico (células da glia e neurônio), células de revestimento (interno e externo) do encéfalo (meninge e epêndima) e as de origem vascular como o plexo coroide. As secundárias foram as metastáticas ou que afetaram o encéfalo por extensão local (Dewey et al. 2016a).

O tempo de evolução clínica foi determinado a partir do início dos sinais encontrados no exame neurológico até o momento da morte ou eutanásia dos cães. Cães submetidos à eutanásia por opção dos proprietários ou que morreram em decorrência da doença e de suas complicações (síndrome paraneoplásica) foram encaminhados para necropsia.

\section{RESULTADOS E DISCUSSÃO}

Com base nos critérios pré-estabelecidos, 40 cães foram incluídos nessa pesquisa (Quadro1). Destes, 10 cães com tumores encefálicos também foram envolvidos no estudo de Santos et al. (2012), sendo dois astrocitomas, três meningiomas, dois ependimomas, um oligodendroglioma, um craniofaringioma e um adenocarcinoma prostático. Porém, os autores envolveram tanto tumores do encéfalo como da medula espinhal. Por outro lado, nesta pesquisa foi feita uma análise somente dos tumores encefálicos e sua classificação (primário e secundário), bem como a inclusão de exames de auxilio ao diagnóstico como hemograma, bioquímica sérica, urina e do LCE, cujos achados complementaram as informações obtidas por Santos et al. (2012).

Quanto ao sexo, 22 (55\%) cães eram fêmeas e 18 (45\%) eram machos. Mesmo havendo uma maior prevalência em cadela, não se pode afirmar, neste estudo, que as fêmeas foram as mais acometidas, corroborando com as afirmações obtidas por Snyder et al. (2006) e Sturges et al. (2008) que descreveram a ausência de predisposição quanto ao gênero nos cães acometidos por neoplasias intracranianas. Em um estudo desenvolvido por Hsu et al. (1997), foi verificado que mulheres com meningiomas apresentavam maior número de receptores para progesterona, o que justifica possivelmente a maior prevalência desse neoplasma em mulheres. Neste estudo, nove cadelas tinham meningiomas e cinco delas eram castradas, mesmo assim, impede em afirmar a relação hormonal com as neoplasias encefálicas na espécie canina.

Quanto à raça, os cães sem raça definida foram os mais acometidos (14/40 (35\%), seguidos pelo Boxer $(11 / 40$ (27,5\%), 
Quadro 1. Representação quanto ao tipo e origem da neoplasia, sexo, idade, raça, localização e tempo de evolução (média) de 40 cães com diagnóstico de neoplasias encefálicas (primárias e secundárias) atendidos no Hospital Veterinário Universitário da Universidade Federal de Santa Maria

\begin{tabular}{|c|c|c|c|c|c|c|}
\hline Tipo de neoplasia (n) & Origem & Sexo (n) & Idade média (anos) & Raça (n) & $\begin{array}{c}\text { Localização } \\
\text { (n) }\end{array}$ & $\begin{array}{l}\text { Evolução clínica } \\
\text { em dias (média) }\end{array}$ \\
\hline \multirow[t]{5}{*}{ Meningioma (13) } & $P$ & $M(4)$ & 10,8 & Boxer (5) & TC (6) & 56,4 \\
\hline & & $F(9)$ & & SRD (4) & TE (1) & \\
\hline & & & & $\mathrm{PA}(2)$ & $\mathrm{C}(2)$ & \\
\hline & & & & Dachshund (1) & MF (4) & \\
\hline & & & & Poodle (1) & & \\
\hline \multirow[t]{3}{*}{ Oligodendroglioma (5) } & $\mathrm{P}$ & M (3) & 9,5 & Boxer (3) & $\mathrm{TC}(4)$ & 61 \\
\hline & & $\mathrm{F}(2)$ & & Bulldog inglês(1) & MF (1) & \\
\hline & & & & Pit Bull (1) & & \\
\hline \multirow[t]{3}{*}{ Astrocitoma (4) } & $\mathrm{P}$ & M (3) & 10 & SRD (2) & TC (3) & 37,7 \\
\hline & & $\mathrm{F}(1)$ & & Boxer (1) & TE (1) & \\
\hline & & & & Labrador retriever (1) & & \\
\hline \multirow[t]{3}{*}{ Ependimoma (3) } & $P$ & M (2) & 11 & Boxer (2) & $\mathrm{TC}(1)$ & 34,6 \\
\hline & & $\mathrm{F}(1)$ & & SRD (1) & TE (1) & \\
\hline & & & & & $\mathrm{C}(1)$ & \\
\hline \multirow[t]{2}{*}{ Papiloma de plexo coroide (1) } & $P$ & $\mathrm{M}(0)$ & 10 & SRD (1) & MF (1) & 60 \\
\hline & & $\mathrm{F}(1)$ & & & & \\
\hline \multirow[t]{2}{*}{ Carcinoma de plexo coroide (1) } & $\mathrm{P}$ & $\mathrm{M}(0)$ & 10 & SRD (1) & MF (1) & 60 \\
\hline & & $\mathrm{F}(1)$ & & & & \\
\hline \multirow[t]{2}{*}{ Carcinoma cromófobo de pituitária (1) } & S & $\mathrm{M}(0)$ & 11 & Yorkshire terrier (1) & $\mathrm{TC}(1)$ & 90 \\
\hline & & $\mathrm{F}(1)$ & & & & \\
\hline \multirow[t]{2}{*}{ Hemangiossarcoma cutâneo (3) } & S & M (1) & 9,6 & Pit Bull (2) & $\mathrm{TC}(2)$ & 13,3 \\
\hline & & $\mathrm{F}(2)$ & & SRD (1) & $\mathrm{C}(1)$ & \\
\hline \multirow[t]{2}{*}{ Carcinoma mamário (2) } & S & $\mathrm{M}(0)$ & 12,5 & Dachshund (1) & $\mathrm{TC}(1)$ & 14,5 \\
\hline & & $\mathrm{F}(2)$ & & SRD (1) & MF (1) & \\
\hline \multirow[t]{2}{*}{ Adenocarcinoma prostático (1) } & $S$ & M (1) & 14 & SRD (1) & $\mathrm{TC}(1)$ & 30 \\
\hline & & $\mathrm{F}(0)$ & & & & \\
\hline \multirow[t]{2}{*}{ Osteossarcoma (2) } & S & M (1) & 12,5 & Poodle (1) & $\mathrm{TC}(2)$ & 13,5 \\
\hline & & $\mathrm{F}(1)$ & & Collie (1) & & \\
\hline \multirow[t]{2}{*}{ TMBNP (2) } & $S$ & M (1) & 9,5 & Labrador retriever (1) & $\mathrm{TE}(2)$ & 14,5 \\
\hline & & $\mathrm{F}(1)$ & & SRD (1) & & \\
\hline \multirow[t]{2}{*}{ Craniofaringioma (1) } & S & $\mathrm{M}(0)$ & 6 & Airedale terrier (1) & MF (1) & 10 \\
\hline & & $\mathrm{F}(1)$ & & & & \\
\hline \multirow[t]{2}{*}{ Carcinoma de células escamosas (1) } & S & M (1) & 14 & SRD (1) & TC (1) & 90 \\
\hline & & $F(0)$ & & & & \\
\hline
\end{tabular}

$\mathrm{P}=$ Primária, $\mathrm{S}=$ secundária, $\mathrm{M}=$ macho, $\mathrm{F}=$ fêmea, $\mathrm{SRD}=$ sem raça definida, $\mathrm{PA}=$ Pastor alemão, $\mathrm{TC}=$ tálamo córtex $\mathrm{TE}=$ tronco encefálico, $\mathrm{C}=$ cerebelo, $\mathrm{MF}=$ multifocal, TMBNP = tumor maligno da bainha de nervo periférico (trigêmeo).

Pit bull (3/40 (7,5\%), Dachshund (2/40 (5\%), Pastor alemão $(2 / 40(5 \%)$, Poodle $(2 / 40(5 \%)$, Labrador retriever $(2 / 40$ $(5 \%)$, Airedale terrier $(1 / 40(2,5 \%)$, Bulldog inglês $(1 / 40$ $(2,5 \%)$, Collie $(1 / 40$ (2,5\%) e Yorkshire terrier $(1 / 40$ (2,5\%). Esses resultados coincidem com os achados de Snyder et al. (2006) e Song et al. (2013)que também relataram cães sem raça definida como os mais acometidos, porém, os autores deste trabalho não podem afirmar que os mesmos foram os mais prevalentes, haja vista que, não há um estudo demonstrando quais as raças mais atendidas pela Instituição durante o período do estudo. Por outro lado, Sturges et al. (2008) mencionaram que Golden retriever, Boxer e Schnauzer miniatura estão entre os mais acometidos, sugerindo a hipótese de predisposição racial. Quanto à idade, os cães afetados variaram de seis a 14 anos, com média de dez anos e seis meses de idade. Resultado semelhante ao mencionado por Bagley et al. (1999) e Snyder et al. (2006) e superior ao descrito por Song et al. (2013) que a média de idade foi de sete anos de idade.

Em relação aos sinais clínicos, foi verificado crises epilépticas generalizadas (16/40 (40\%), alteração comportamental (agressividade, andar compulsivo) (15/40 (37,5\%), síndrome vestibular central (presença de inclinação de cabeça associada à deficiência proprioceptiva e/ou sonolência) (12/40 (30\%), andar em círculo (8/40 (20\%), alteração no nível de consciência (sonolência) (6/40 (15\%), andar compulsivo (5/40 (12,5\%), tetraparesia não ambulatória $(5 / 40(10,8 \%)$, ataxia cerebelar (hipermetria associada à postura de base ampla e/ou tremor de 
intenção) (4/40 (10\%), dificuldade de deglutição (3/40 (7,5\%), aumento de volume no crânio $(3 / 40(7,5 \%)$, hiperestesia cervical (2/40 (5\%) e amaurose (cegueira cortical) (2/40 (5\%). Esses resultados foram semelhantes ao estudo de Bagley et al. (1999) e Snyder et al. (2006) em que descreveram a crise epiléptica como a manifestação neurológica mais frequente em cães com neoplasias encefálicas. Vale salientar que cães com neoplasias encefálicas apresentando crises epilépticas podem inicialmente não apresentar alteração no exame neurológico, levando, muita das vezes, o diagnóstico equivocado de epilepsia primária ou idiopática. Por isso, sempre considerar que as crises epilépticas têm uma causa determinante no momento da abordagem e indicar a realização de imagens do encéfalo pela tomografia computadorizada ou ressonância magnética para tentar excluir os tumores como causa.

Assim como no estudo de Snyder et al. (2006), a mudança comportamental (agressividade e andar compulsivo) foi o segundo sinal clínico encontrado e provavelmente seja ainda mais comum, visto que, pode passar desapercebido pelos proprietários e clínicos devido a dificuldade inicial em identificar, principalmente quando as alterações são menos severas. Costa (2009) recomendou uma anamnese minuciosa e detalhada para identificar essas mudanças comportamentais menos evidentes com o intuito de evitar que elas não sejam detectadas.
Quanto às regiões encefálicas, a tálamo-cortical (55\% (22/40) foi a mais acometida, seguida da tronco-encefálica (12,5\% (5/40) e do cerebelo (10\% (4/40). Localização multifocal foi observada em 22,5\% dos casos (9/40). Esses resultados corroboraram com os já descritos previamente em outras pesquisas (Montoliu et al. 2006, Snyder et al. 2006, 2008, Sturges et al. 2008). Segundo Snyder et al. $(2006,2008)$ e Sturges et al. (2008), assim como observado neste estudo, na maioria dos casos, os sinais neurológicos foram compatíveis com lesão focal, porém sinais multifocais podem ser observados ocasionalmente em neoplasias que ocupam mais de uma região anatômica (Snyder et al. 2006, Snyder et al. 2008, Sturges et al. 2008, Costa 2009).

As neoplasias de origem primária foram as mais encontradas neste estudo e perfizeram 67,5\% dos casos (27/40), sendo o meningioma (48,1\% (13/27) o mais comum, seguido do oligodendroglioma (18,5\% (5/27), astrocitoma $(14,8 \%(4 / 27)$, ependimoma $(11,1 \%(3 / 27)$, papiloma de plexo coroide $(3,7 \%(1 / 27)$, carcinoma de plexo coroide $(3,7 \%$ (1/27) (Fig.1A-D). Uma análise de 400 casos de neoplasias encefálicas primárias em cães demonstrou que 190 (47,5\%) corresponderam ao meningioma, seguido de 76 (19\%) oligodendrogliomas, $54(13,5 \%)$ astrocitomas e $24(6 \%)$ tumores de plexo coroide (Snyder et al. 2006, Song et al.
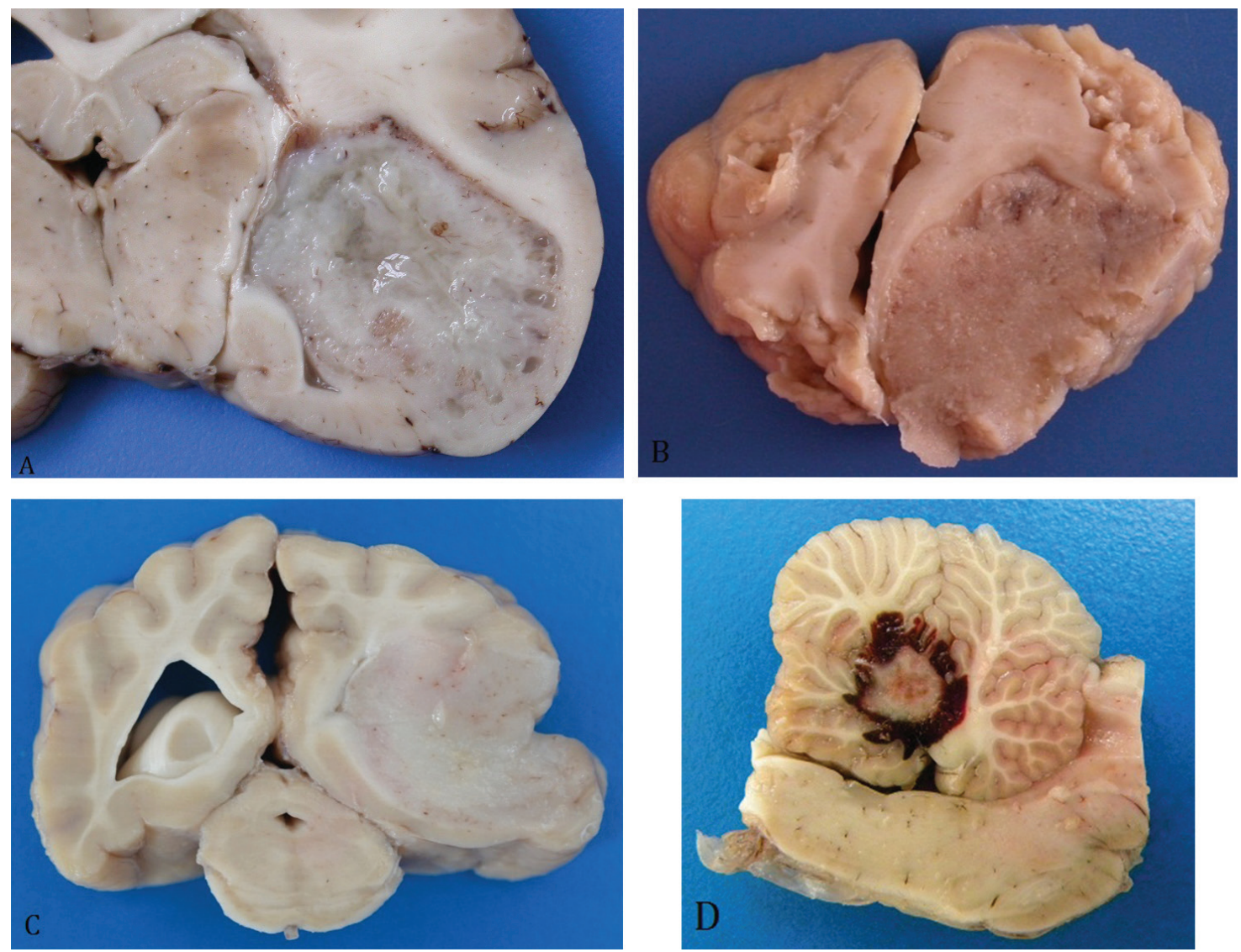

Fig.1. Neoplasia encefálica em cão. (A) Oligodendroglioma substituindo o parênquima do lobo temporal com deslocamento do tálamo direito. (B) Meningioma na região do bulbo olfatório esquerdo. (C) Oligodendroglioma substituindo o parênquima do lobo temporal, comprimindo e deslocando o tálamo, o mesencéfalo e o hipocampo. Esse tumor não foi observado durante exame de tomografia computadorizada. (D) Nódulo branco-avermelhado, circundado por um halo vermelho no cerebelo em decorrência de metástase de hemangiossarcoma cutâneo. 
2013). Embora Costa (2009) e Dewey (2016) descreveram alta incidência de meningiomas e gliomas nas raças dolicocefálicas e braquiocefálicas, respectivamente, no presente estudo, não se observou diferença nessa prevalência nos 11 cães braquiocefálicos e nos três dolicocefálicos. Uma observação interessante foi o Boxer, uma raça braquiocefálica que foi acometida pelas principais neoplasias primárias encontradas nesse levantamento (Quadro 1).

0 acometimento encefálico por neoplasmas se dá por extensão direta (invasão) ou por via hematógena. De modo geral, carcinomas fazem metástase para o sistema nervoso central (SNC) formando um nódulo tumoral encefálico. Por outro lado, sarcomas costumam fazer metástases para estruturas adjacentes, como osteossarcoma para os ossos do crânio. A exceção é o hemangiossarcoma, que em geral, faz metástase direta para o encéfalo (Costa 2009). No presente estudo, dos 40 casos, 13 (32,5\%) apresentaram neoplasias secundárias, sendo seis metastáticos (três hemangiossarcomas cutâneos, dois carcinomas mamários e um adenocarcinoma prostático) e sete infiltrativos (dois osteossarcomas, dois tumores maligno da bainha de nervo periférico afetando o nervo trigêmeo, um craniofaringioma, um carcinoma de células escamosas e um carcinoma cromófobo de hipófise). Em dois estudos sobre neoplasias encefálicas secundárias (Snyder et al. 2008, Song et al. 2013), de 385 cães, 124 (32,2\%) apresentaram hemangiossarcoma, 68 (17,7\%) carcinoma de pituitária, 62 (16\%) linfoma e $60(15,5 \%)$ carcinoma metastático. Os autores desse trabalho ressaltam a importância do exame clínico geral em busca de tumores cutâneos e de exames complementares de imagem para tumores intracavitários (abdome e tórax) quando se inclui as neoplasias no diagnóstico diferencial e, uma vez detectado, indicar a biópsia (incisional ou excisional) e análise histopatológica, independente do tamanho da massa tumoral com o intuito de reforçar o diagnóstico e a possível origem da neoplasia encefálica.

Quanto ao LCE, existe controvérsia sobre o valor da análise em pacientes com suspeita de neoplasia encefálica, visto que, a coleta pode gerar herniações cerebrais em decorrência do aumento de pressão intracraniana (Costa 2009, Dewey 2016). Essa alteração foi observada em apenas um paciente do presente estudo e confirmada na necropsia. Diante disso, embora não realizada em todos os casos devido à limitação de custos por parte dos tutores, recomenda-se tomografia computadorizada ou ressonância magnética antes da coleta de LCE e, na presença de imagens sugestivas de neoplasias, deve-se evitar o procedimento, visto que, os resultados raramente confirmam o diagnóstico. Embora alterações do LCE sejam inespecíficas (Dickinson et al. 2006, Costa 2009, Di Terlizzi \& Platt 2009), em apenas $10 \%$ dos cães com neoplasias intracranianas é normal (Costa 2009). A alteração mais comumente observada é a dissociação albuminocitológica, alteração em que a concentração de proteína total está elevada, sem aumento correspondente do número de leucócitos (Dickinson et al. 2006, Costa 2009). Outra alteração observada é a pleocitose neutrofílica (Dickinson et al. 2006, Costa 2009), no entanto, essa alteração no LCE também pode ser encontrada em outras doenças neurológicas, como encefalites infecciosas ou não infecciosas (Di Terlizzi \& Platt 2009). No presente estudo, dos 24 cães em que foram realizadas coleta e análise do LCE, $16(66,6 \%)$ apresentaram dissociação albuminocitológica, dois $(8,3 \%)$ pleocitose linfocítica com aumento de proteína e um $(4,1 \%)$ pleocitose mista e aumento de proteína. Em cinco cães $(20,8 \%)$ não foram observadas alterações (Quadro 2).

Na necropsia, dos 27 cães com neoplasias primárias, dez (37\%) apresentaram diferentes tipos de neoplasias em outras regiões não encefálicas como pulmão, glândulas mamárias, vesícula urinária, linfonodo, pâncreas, ovário e rim. Porcentagem superior à relatada por Snyder et al. (2006), na qual apenas $6 \%$ dos cães apresentaram dois ou mais tipos diferentes de neoplasias independentes identificadas na necropsia. No presente estudo, apenas um cão apresentou neoplasia primária e secundária concomitante no SNC, ou seja, um meningioma encefálico e uma metástase de carcinoma mamário para medula espinhal cervical. Em relação aos 13 cães com neoplasias secundárias, o mesmo tumor que se encontrava no

Quadro 2. Resultados do líquido cerebroespinhal (LCE) de 24 cães com neoplasias encefálicas (primárias e secundárias) atendidos no Hospital Veterinário Universitário da Universidade Federal de Santa Maria

\begin{tabular}{|c|c|}
\hline Tipo de neoplasia (n) & Alterações no LCE \\
\hline Meningioma (7) & Dissociação albuminocitológica* \\
\hline Meningioma (3) & Sem alteração \\
\hline Meningioma (1) & Pleocitose linfocítica ( $>50 \%$ linfócitos) e aumento de proteínas \\
\hline Astrocitoma (1) & Dissociação albuminocitológica \\
\hline Ependimoma (1) & Dissociação albuminocitológica \\
\hline Oligodendroglioma (1) & Dissociação albuminocitológica \\
\hline Oligodendroglioma (1) & Pleocitose linfocítica (>50\% linfócitos) e aumento de proteínas \\
\hline Oligodendroglioma (1) & Sem alteração \\
\hline Papiloma de plexo coroide (1) & Dissociação albuminocitológica \\
\hline Craniofaringioma (1) & Pleocitose mista (50\% linfócito e 50\% neutrófilo) e aumento de proteínas \\
\hline Adenocarcinoma próstata (1) & Dissociação albuminocitológica \\
\hline Carcinoma mamário (1) & Dissociação albuminocitológica \\
\hline Hemangiossarcoma (2) & Dissociação albuminocitológica \\
\hline Hemangiossarcoma (1) & Sem alteração \\
\hline TMBNP (1) & Dissociação albuminocitológica \\
\hline
\end{tabular}

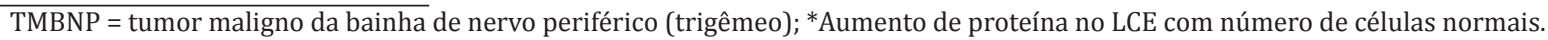


SNC, foi encontrado também na pele (25\%), no pulmão (25\%), no coração (25\%), no rim (25\%), na adrenal (25\%), na glândula mamária $(16,7 \%)$, na próstata $(8,3 \%)$ e na tonsila palatina $(8,3 \%)$. Resultado semelhante ao observado por Snyder et al. (2008), em que o pulmão e os rins foram os mais acometidos, além do SNC. Diante disso, antes de submeter o paciente a exames mais específicos, como tomografia computadorizada e ressonância magnética, indica-se a realização de exames complementares que incluem hemograma, bioquímica sérica (função hepática e renal), urinálise, radiografias torácicas (no mínimo em duas incidências) e ultrassonografia abdominal, na busca de lesões metastáticas e/ou outras neoplasias (Costa 2009). Nesse trabalho, no entanto, os exames hematológicos, bioquímicos e de urina encontravam-se dentro dos parâmetros de referência para a espécie.

Com relação aos exames de imagens, a tomografia computadorizada e a ressonância magnética são as mais indicadas para o diagnóstico de neoplasias encefálicas (Morozumi et al. 1997, Kraft \& Gavin 1999, Dewey et al. 2016a). Porém, algumas limitações podem ser observadas em ambas as modalidades, sendo, na tomografia computadorizada, quando a região a ser explorada é a fossa caudal, devido ao efeito de endurecimento de feixe (beam hardening) em decorrência da densidade da porção petrosa do osso temporal. Nesse estudo, um cão Boxer que apresentava oligodendroglioma no lobo temporal e visivelmente observado no exame macroscópico durante a necropsia, não foi identificado pela tomografia computadorizada, mesmo com injeção de contraste, possivelmente pela explicação dos autores acima mencionados (Fig.1C). Outra justificativa seria pelo crescimento lento dos tumores como os gliomas infiltrativos que podem não ser detectados pela tomografia computadorizada, principalmente quando houver pouco ou nenhum preenchimento de contraste devido à impermeabilidade da vascularização local intacta (Kraft \& Gavin 1999).

O tempo de evolução clínica foi determinado a partir do início dos sinais encontrados no exame neurológico até o momento da morte ou eutanásia dos cães, sendo progressiva em todos os pacientes. Os tumores de origem primária apresentaram média de tempo de evolução de 53 dias, enquanto os de origem secundária apresentaram uma evolução mais rápida (média de 20,4 dias). Uma provável explicação na diferença entre o tempo de evolução pode ser pelas características histológicas dos tumores, geralmente benigno e de evolução lenta nas neoplasias primárias e mais agudas nas secundárias. Entre os tumores de origem primária de evolução lenta (meses a anos), pode-se citar o meningioma, que representou $48 \%$ dos tumores primários deste estudo. Heidner et al. (1991) também verificaram que a maioria dos cães com evolução lenta dos sinais neurológicos tinha meningioma. Por outro lado, as neoplasias secundárias e os tumores primários malignos apresentaram uma progressão mais rápida dos sinais, sendo associado ao grande número de regiões encefálicas acometidas e pelo envolvimento simultâneo extra e intracraniano. 0 tempo de evolução dos sinais deste estudo foi superior aos obtidos por Snyder et al. (2006), em que a média de sobrevida para 173 cães com neoplasias primárias foi de 26 dias e semelhante ao estudo de Snyder et al. (2008), que a média foi de 21 dias para 177 cães com neoplasias secundárias.

A relevância clínica desse estudo está na representação dos diferentes tumores que afetam o encéfalo de cão, seja de origem primária ou secundária e, uma atenção especial aos sinais neurológicos, tendo a crise epiléptica como principal manifestação encontrada, revelando ao clínico da importância de achar as causas e tentar evitar, inicialmente, do diagnóstico de epilepsia primária ou idiopática.

\section{CONCLUSÕES}

Com base nos resultados encontrados a partir dos registros médicos do SN-HVU, pode se concluir que neoplasias encefálicas acometem diferentes raças, sendo o Boxer afetado por todos os tipos de tumores primários e a idade predominante foi superior a seis anos.

Os achados no hemograma, bioquímica sérica e urinálise não auxiliaram na suspeita da doença, diferentemente do líquido cerebroespinhal (LCE), cuja dissociação albuminocitólogica foi a alteração mais observada.

0 tempo de evolução dos sinais clínicos é maior para as neoplasias primárias, sendo a crise epiléptica e a mudança de comportamento, independente da evolução (crônica ou aguda) as alterações mais observadas.

\section{REFERÊNCIAS}

Bagley R.S., Gavin P.R., Moore M.P., Silver G.M., Harrington M.L. \& Connors R.L. 1999. Clinical signs associated with brain tumors in dogs: 97 cases (1992-1997). J. Am. Vet. Med. Assoc. 215(6):818-819. PMid:10496135.

Bley C.R., Sumova A., Roos M. \& Kaser-Hotz B. 2005. Irradiation of brain tumors in dogs with neurologic disease. J. Vet. Intern. Med. 19(6):849-854. http://dx.doi.org/10.1111/j.1939-1676.2005.tb02776.x. PMid:16355679.

Costa R.C. 2009. Neoplasias do sistema nervoso, p.412-427. In: Daleck R.C., Nardi A.B. \& Rodaski S. (Eds), Oncologia em Cães e Gatos. Roca, São Paulo.

Dewey C.W. 2016. Encephalopathies: disorders of the brain, p.141-236. In: Dewey C.W. \& Costa R.C. (Eds), A Pratical Guide to Canine and Feline Neurology. 3rd ed. Blackwell Plubishing, Iowa.

Dewey C.W., Costa R. \& Ducoté J.M. 2016a. Neurodiagnostics, p.9-28. In: Dewey C.W. \& Costa R.C. (Eds), A Pratical Guide to Canine and Feline Neurology. 3rd ed. Blackwell Plubishing, Iowa.

Dewey C.W., Costa R. \& Thomas W. 2016b. Perfoming the neurologic examination, p.61-86. In: Dewey C.W. \& Costa R.C. (Eds), A Pratical Guide to Canine and Feline Neurology. 3rd ed. Blackwell Plubishing, Iowa.

Di Terlizzi R. \& Platt S.R. 2009. The function, composition and analysis of cerebrospinal fluid in companion animals. II. Analysis. Vet. J. 180(1):15-32. http://dx.doi.org/10.1016/j.tvjl.2007.11.024. PMid:18294880.

Dickinson P.J., Sturges B.K., Kass P.H. \& Lecouteur R.A. 2006. Characteristics of cisternal cerebrospinal fluid associated with intracranial meningiomas in dogs: 56 cases (1985-2004). J. Am. Vet. Med. Assoc. 228(4):564-567. PMid:16478433.

Heidner G.L., Kornegay J.N., Page R.L., Dodge R.K. \& Thrall D.E. 1991. Analysis of survival in a retrospective study of 86 dogs with brain tumors. J. Vet. Intern. Med. 5(4):219-226. http://dx.doi.org/10.1111/j.1939-1676.1991. tb00952.x. PMid:1941756.

Hsu D.W., Efird J.T. \& Hedley-Whyte E.T. 1997. Progesterone and estrogen receptors in meningioma. Prognostic considerations. J. Neurosurg. 86(1):113120. http://dx.doi.org/10.3171/jns.1997.86.1.0113. PMid:8988089.

Kraft S.L. \& Gavin P.R. 1999. Intracranial neoplasia. Clin. Tech. Small Anim. Pract. 14(2):112-123. http://dx.doi.org/10.1016/S1096-2867(99)800097. PMid:10361361

Kraft S.L., Gavin P.R., Dehaan C., Moore M., Wendling L.R. \& Leathers C.W. 1997. Retrospective review of 50 canine intracranial tumors evaluated by 
magnetic resonance imaging. J. Vet. Intern. Med. 11(4):218-225. http:// dx.doi.org/10.1111/j.1939-1676.1997.tb00094.x. PMid:9298476.

Marcasso R.A., Moreira J.R., Valentim L.G., Arias M.V.B. \& Bracarense A.P.F.R.L. 2015. Meningiomas em cães: aspectos clínicos, histopatológicos e imunohistoquímicos. Pesq. Vet. Bras. 35(10):844-852. http://dx.doi.org/10.1590/ S0100-736X2015001000005.

Mcentee M.C. \& Dewey C.W. 2013. Tumors of the nervous system, p.583-596. In: Withrow S.J., Vail D.M. \& Page R.L. (Eds), Withrow and MacEwen's Small Animal Clinical Oncology. 5th ed. W.B. Saunders, Philadelphia. http:// dx.doi.org/10.1016/B978-1-4377-2362-5.00030-X.

Montoliu P., Anor S., Vidal E. \& Pumarola M. 2006. Histological and immunohistochemical study of 30 cases of canine meningioma. J. Comp. Pathol. 135(4):200-207. http://dx.doi.org/10.1016/j.jcpa.2006.06.006. PMid:17049358.

Morozumi M., Miyahara K., Sato M. \& Hirose T. 1997. Computed tomography and magnetic resonance findings in two dogs and a cat with intracranial lesions. J. Vet. Med. Sci. 59(9):807-810. http://dx.doi.org/10.1292/ jvms.59.807. PMid:9342706.

O’Brien D.P.O. \& Coates J.R. 2010. Brain disease, p.668-725. In: Ettinger S.J. \& Feldman E.C. (Eds), Veterinary Internal Medicine. 7th ed. Elsevier Mosby, Philadelphia.

Ródenas S., Pumarola M., Gaitero L., Zamora A. \& Añor S. 2011. Magnetic resonance imaging findings in 40 dogs with histologically confirmed intracranial tumours. Vet. J. 187(1):85-91. http://dx.doi.org/10.1016/j. tvjl.2009.10.011. PMid:19914851.
Rossmeisl J. \& Pancotto T. 2012. Intracranial neoplasia and secondary pathological effects, p.461-478. In: Platt S. \& Garosi L. (Eds), Small Animal Neurological Emergencies. Manson Publishing, London. http://dx.doi. org/10.1201/b15214-30.

Santos R.P., Fighera R.A., Beckmann D.V., Brum J.S., Ripplinger A., Polidoro Neto D. N., Baumhardt R. \& Mazzanti A. 2012. Neoplasmas envolvendo o sistema nervoso central de cães: 26 casos (2003-2011). Pesq. Vet. Bras. 32(2):153-158. http://dx.doi.org/10.1590/S0100-736X2012000200011.

Snyder J.M., Lipitz L., Skorupski K.A., Shofer F.S. \& Van Winkle T.J. 2008. Secondary intracranial neoplasia in the dog: 177 cases (1986-2003). J. Vet. Intern. Med. 22(1):172-177. http://dx.doi.org/10.1111/j.19391676.2007.0002.x. PMid:18289306.

Snyder J.M., Shofer F.S., Van Winkle T.J. \& Massicotte C. 2006. Canine intracranial primary neoplasia: 173 cases (1986-2003). J. Vet. Intern. Med. 20(3):669675. PMid:16734106

Song R.B., Vite C.H., Bradley C.W. \& Cross J.R. 2013. Postmortem evaluation of 435 cases of intracranial neoplasia in dogs and relationship of neoplasm with breed, age, and body weight. J. Vet. Intern. Med. 27(5):1143-1152. http://dx.doi.org/10.1111/jvim.12136. PMid:23865437.

Sturges B.K., Dickinson P.J., Bollen P.D., Koblik P.D., Kass P.H., Kortz G.D., Vernau K.M., Knipe M.F., Lecouteur R.A. \& Higgins R.J. 2008. Magnetic resonance imaging and histological classification of intracranial meningiomas in 112 dogs. J. Vet. Intern. Med. 22(3):586-595. http://dx.doi.org/10.1111/j.19391676.2008.00042.x. PMid:18466258. 